\title{
A randomized, placebo-controlled pilot trial of armodafinil for fatigue in patients with gliomas undergoing radiotherapy
}

\author{
Eudocia Q. Lee†, Alona Muzikansky', Jan Drappatz', Santosh Kesari, Eric T. Wong, Camilo E. Fadul, \\ David A. Reardon, Andrew D. Norden, Lakshmi Nayak, Mikael L. Rinne, Brian M. Alexander, Nils D. Arvold, \\ Lisa Doherty, Jennifer Stefanik, Debra LaFrankie, Sandra F. Ruland, Julee Pulverenti, Katrina H. Smith, \\ Sarah C. Gaffey, Samantha Hammond, and Patrick Y. Wen
}

Dana-Farber/Brigham and Women's Cancer Center, Boston, Massachusetts (E.Q.L., D.A.R., A.D.N., L.N., M.L.R., B.M.A., N.D.A., L.D., J.S., D.L., S.F.R., J.P., K.H.S., S.C.G., S.H., P.Y.W.); Harvard Medical School, Boston, Massachusetts (E.Q.L., E.T.W., D.A.R., A.D.N., L.N., M.L.R., B.M.A., N.D.A., P.Y.W.); Massachusetts General Hospital, Boston, Massachusetts (A.M.); University of Pittsburgh, Pittsburgh, Pennsylvania (J.D.); University of California San Diego, La Jolla, California (S.K.); Beth Israel Deaconess Medical Center, Boston, Massachusetts (E.T.W.); Dartmouth Hitchcock Medical Center, Lebanon, New Hampshire (C.E.F.); Geisel School of Medicine at Dartmouth, Hanover, New Hampshire (C.E.F.)

Corresponding Author: Eudocia Q. Lee, MD, MPH, Center for Neuro-Oncology, Dana Farber Cancer Institute, 450 Brookline Avenue, Dana 2110, Boston, MA 02215 (eqlee@partners.org).

†These authors contributed equally to the work.

See the editorial by Grant and Brown, on pages 759-760.

Background. Fatigue is common among glioma patients undergoing radiotherapy (RT) and impacts quality of life (QOL). We evaluated whether armodafinil, a wakefulness-promoting medication, improves fatigue in glioma patients undergoing RT.

Methods. Eligibility criteria included age $\geq 18$ years, Karnofsky performance status $\geq 60$, and grade $2-4$ glioma undergoing RT to a total dose of 50-60 Gy. Patients were randomized 1:1 to armodafinil or placebo for 8 weeks beginning within 10 days of starting RT. Fatigue and QOL were assessed at baseline, day 22, day 43, and day 56 with the Functional Assessment of Chronic Illness Therapy - Fatigue (FACIT-F), the Functional Assessment of Cancer Therapy - General (FACT-G), the Brief Fatigue Inventory (BFI), and the Cancer Fatigue Scale (CFS). The primary aim was to detect a difference in the 42-day change in FACIT-F fatigue subscale between the 2 groups using a 2-sample Wilcoxon statistic.

Results. We enrolled 81 patients total (42 armodafinil and 39 placebo). Armodafinil did not significantly improve fatigue or QOL based on the 42-day change in FACIT-F fatigue subscale, FACT-G, CFS, or BFI. Further analysis suggests no difference between the arms even after accounting for the potential bias of missing data. Treatment was well tolerated with few grade 3 or 4 toxicities.

Conclusions. While treatment was well-tolerated, an 8-week course of armodafinil did not improve fatigue or QOL in glioma patients undergoing RT in this pilot study. Further studies are needed to determine whether pharmacologic treatment improves fatigue in glioma patients undergoing RT.

Keywords: armodafinil, clinical trial, fatigue, glioblastoma, glioma.

Fatigue is a common symptom in primary brain tumor patients, with $40 \%-70 \%$ reporting fatigue during the course of their illness. ${ }^{1}$ The prevalence of fatigue is even higher in primary brain tumor patients undergoing cranial irradiation, with $>80 \%$ of patients reporting fatigue during radiation (RT). ${ }^{2}$ Only a few randomized studies evaluating pharmacologic and/or nonpharmacologic interventions for fatigue have been performed in cancer patients, especially glioma patients. ${ }^{3}$ One recent randomized study demonstrated that armodafinil did not improve fatigue in participants with primary brain tumors (including gliomas) undergoing RT. ${ }^{4}$ However, those with greater baseline fatigue did experience improved quality of life (QOL) and reduced fatigue when using armodafinil.

Armodafinil is an oral wakefulness-promoting medication that is the R-enantiomer of modafinil. It is approved by the United States Food and Drug Administration (FDA) to improve 
wakefulness in adult patients with excessive sleepiness associated with obstructive sleep apnea, narcolepsy, and shift work disorder. Its precise mechanism is unknown, but its pharmacologic profile is distinct from that of sympathomimetic agents. We performed a randomized, placebo-controlled pilot trial of armodafinil for fatigue in participants with gliomas undergoing RT.

\section{Materials and Methods}

\section{Patients}

Patients aged $\geq 18$ years with Karnofsky Performance Status (KPS) $\geq 60$ and a grade $2-4$ glioma who were scheduled to receive RT to a total dose of 50-60 Gy were eligible. Concurrent chemotherapy was allowed. Exclusion criteria included the use of psychostimulants within the past 30 days or a history of severe depression, psychotic disorder, bipolar disorder, anxiety disorder, Tourette's syndrome, significant cardiac arrhythmia, or unstable angina. Because outcomes were based on patientreported questionnaires, participants were required to be able to read and respond to questions in English. Approval from institutional review boards and/or independent ethics committees was obtained at each site. All patients provided written, informed consent. This study was registered on clinicaltrials.gov (NCT00766467).

\section{Treatment and Study Design}

This was a randomized, placebo-controlled, multicenter pilot study that enrolled patients between August 2008 and April 2014 (CONSORT diagram; Fig. 1). Participants were randomly assigned $1: 1$ at registration to receive armodafinil $150 \mathrm{mg}$ or placebo daily for 56 days, starting within 10 days from the start of RT. Endpoints for fatigue relief and QOL were assessed by the use of several questionnaires obtained at baseline (prior to starting treatment), day 22 , day 43 , and day 56 . The questionnaires included the Functional Assessment of Chronic Illness Therapy - Fatigue (FACIT-F), the Functional Assessment of Cancer Therapy - General (FACT-G), the Brief Fatigue Inventory (BFI), the Cancer Fatigue Scale (CFS), and the Beck Depression Inventory (BDI).

FACIT-F is a well-validated QOL instrument widely used for the assessment of cancer-related fatigue in clinical trials. ${ }^{5}$ It consists of the 27-item FACT-G (which assesses QOL based on physical, social/family, emotional, and functional well-being) and the 13-item FACIT-F fatigue subscale (which assesses the impact of fatigue on daily activities). Each item is assessed on a 5 -point Likert scale ( $0=$ not at all to $4=$ very much). By scoring convention, after appropriate reversal scoring of 11 items, the FACIT- $F$ fatigue subscale (FACIT-fatigue) score ranges from 0 to 52 (lower score indicating more fatigue). A score $<30$ indicates severe fatigue. We chose the FACIT-fatigue scale as the primary outcome measure because it has been widely used in trials of cancer-related fatigue ${ }^{1,6}$ and has good internal consistency and test-retest reliability. ${ }^{7}$ CFS is a 15 -item instrument with 3 subscales to estimate physical, cognitive, and activity-related fatigue. $^{8}$ The scale is also assessed on a 5 -point Likert scale $(0=$ not at all to $4=$ very much) giving a total score ranging from 0 to 60 (higher score indicating more fatigue). BFI is a 9 -item instrument used to assess fatigue on a rating scale from 0 to 10, with higher scores indicating more fatigue. The scale has been validated in diverse cancer populations. ${ }^{9}$

Because of the minimal expected toxicities with this FDA-approved drug, clinic visits were required only for screening, baseline assessments, receiving orientations and study drug, and at the end of the study; remaining assessments occurred by telephone and by completing documents (questionnaires and the pill diary) at home. Participants were monitored and remained on the study for 8 weeks. Armodafinil was supplied by Cephalon, Inc., a wholly owned subsidiary of Teva Pharmaceuticals.

\section{Statistical Analyses}

The primary endpoint was the difference in the 42-day change (baseline vs day 43) in FACIT-fatigue between the 2 treatment groups. This time point was selected as the primary endpoint because it coincided with the end of radiation in most patients. With 60 participants, the study had $80 \%$ power to detect a difference of 8.076 points in FACIT-fatigue with a 2-tailed type I error of 0.1 . Assuming $25 \%$ attrition, planned enrollment was 80 participants. Interim analysis was based on the O'BrienFleming group sequential boundaries method and treatment groups compared by the Wilcoxon rank-sum test. Primary analysis included participants with complete or near complete baseline and day 43 data irrespective of the amount of treatment received. Complete study treatment was defined per protocol as having missed no more than 6 of the 56 scheduled doses.

Several methods were used to test the difference between the 2 arms in the presence of missing data, assuming various forms of missingness. Under the missing completely at random (MCAR) assumption, a single linear mixed model with random intercept and trend was fitted, explaining the change in FACIT-fatigue at day 43 from baseline as a function of arm. The Standard Multiple Imputation method with missing at random (MAR) assumption was also tested. Under this method, we imputed the missing data (for all 81 randomized participants) based on Markov Chain Monte Carlo (MCMC) method 100 times (thus generating 100 complete datasets). The results from all 100 models were then combined and analyzed using standard inferential statistics. ${ }^{10}$ Lastly, under the assumption that missing data patterns are missing not at random (MNAR), we applied Pattern Mixture models ${ }^{11}$ with Control-Based Pattern Imputation. ${ }^{12,13}$ Implementation of this analysis is similar to the Standard Multiple Imputation approach but incorporates additional steps at the imputation stage that structure the missing data based on the available information in the control group. Further details on all analyses and results are provided in Appendix 1.

Per protocol, interim analysis was performed in April 2011 for safety. Test statistics were calculated for the primary outcomes of change from baseline to day 42 in FACIT-F, FACT-G, CFS, and BFI and compared against the calculated boundaries. Since none of the test statistics for any of the outcomes fell between the predetermined boundaries, enrollment continued to completion. The significance level and power for this interim analysis were 0.058 and 0.68 .

\section{Results}

We enrolled 81 participants ( 42 in the armodafinil arm, 39 in placebo arm) (Table 1). Median ages were 56 years (range 


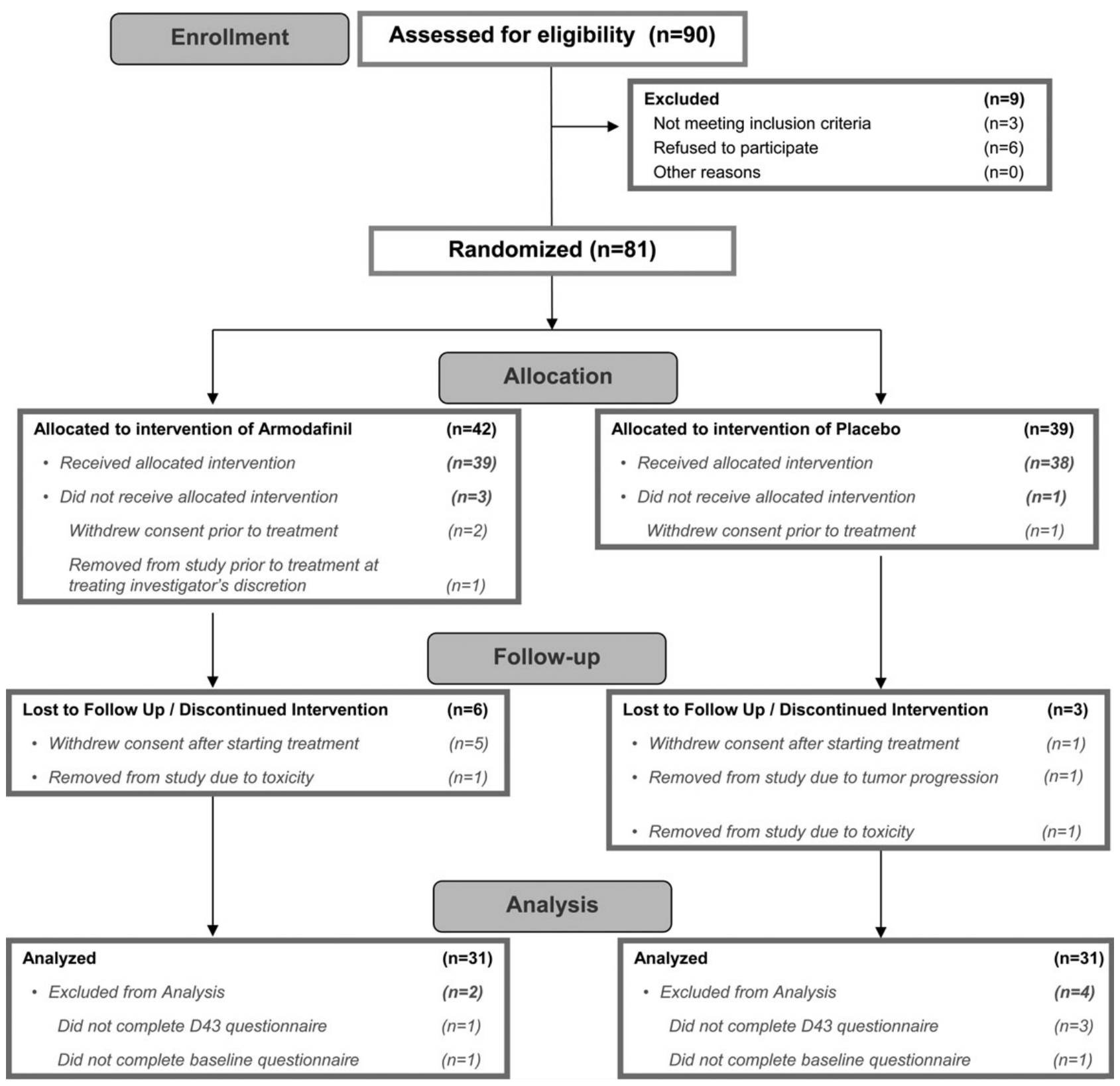

Fig. 1. Consort diagram.

25-79 y) and 54 years (range $19-78$ y) respectively, with median KPS of 90 (range 70-100). All patients had glioma as their histologic diagnosis. Grade 4 was the most common grade in both groups (59.52\% in the armodafinil arm and $47.83 \%$ in the placebo arm). Most participants received concomitant temozolomide $(90.48 \%$ in the armodafinil arm and $92.31 \%$ in the placebo arm). All participants received radiation within the 50-60 Gy range as per eligibility criteria except for 2 participants in the placebo arm (one participant received 62 Gy and the other received $66 \mathrm{~Gy}$ ). There was no significant difference in baseline scores between groups as measured by FACIT-F (total score), FACIT-fatigue, FACT-G, CFS, BFI, or BDI. Median FACIT-fatigue scores at baseline suggest that participants in both arms were experiencing moderate fatigue even prior to starting radiation. Median BDI scores at baseline indicated only mild (if any) depression in most patients.

Of the 42 participants allocated to the armodafinil arm, 31 patients (74\%) had complete or near-complete baseline and day 43 data irrespective of the number of armodafinil doses taken (Fig. 1). Of the 39 participants allocated to the placebo arm, 31 patients (79\%) had complete or near complete baseline and day 43 data irrespective of the number of placebo doses taken (Fig. 1). The proportion of participants with missing data at any time point was not different between arms based on the Fisher exact test. Of the patients analyzed in the armodafinil arm, 27 (87\%) took complete study treatment (defined per protocol as having missed no more than 6 of the 56 scheduled doses). Four patients (13\%) in the armodafinil arm did not 
receive complete treatment for the following reasons: 2 due to noncompliance, one stopped study treatment on day 39 due to grade 2 rash, and one took $50 \mathrm{mg}$ daily as opposed to $150 \mathrm{mg}$

Table 1. Patient characteristics

\begin{tabular}{|c|c|c|}
\hline Characteristic & $\begin{array}{l}\text { Armodafinil } \\
(\mathrm{N}=42)\end{array}$ & $\begin{array}{l}\text { Placebo } \\
(\mathrm{N}=39)\end{array}$ \\
\hline Age (y), median (range) & $56(25-79)$ & $54(19-78)$ \\
\hline Baseline KPS, median (range) & $90(70-100)$ & $90(70-100)$ \\
\hline \multicolumn{3}{|l|}{ Sex, $N(\%)$} \\
\hline Female & $18(42.86 \%)$ & $18(46.15 \%)$ \\
\hline Male & $24(57.14 \%)$ & $21(53.85 \%)$ \\
\hline \multicolumn{3}{|l|}{ Glioma grade, N (\%) } \\
\hline Grade 2 & $1(2.38 \%)$ & $4(10.26 \%)$ \\
\hline Grade 3 & $14(33.33 \%)$ & $12(30.77 \%)$ \\
\hline Grade 4 & $25(59.52 \%)$ & $22(47.83 \%)$ \\
\hline Grade not defined & $2(4.76 \%)$ & $1(2.56 \%)$ \\
\hline $\begin{array}{l}\text { Total dose of radiation, median } \\
\text { (range) }\end{array}$ & 60 Gy $(59.4-60)$ & 60 Gy $(54-66)$ \\
\hline \multicolumn{3}{|l|}{ Concomitant Chemotherapy, N (\%) } \\
\hline Temozolomide & $38(90.48 \%)$ & $36(92.31 \%)$ \\
\hline Temozolomide + bevacizumab & $1(2.38 \%)$ & 0 \\
\hline None & $3(7.14 \%)$ & $3(7.69 \%)$ \\
\hline \multicolumn{3}{|c|}{ Baseline fatigue and QOL, median (range) } \\
\hline FACIT-F (total score) & $80(15-103)$ & $82(25-106)$ \\
\hline FACIT- fatigue subscale score & $38(2-51)$ & $41(8-52)$ \\
\hline FACT-G score & $82(33-106)$ & $86(46-105)$ \\
\hline CFS score & $13.5(0-60)$ & $13.5(0-50)$ \\
\hline BFI score & $2.3(0.1-10)$ & $2.5(0-8.4)$ \\
\hline BDI Score & $7.5(1-20)$ & $7.0(0-23)$ \\
\hline
\end{tabular}

Abbreviations: BDI, Beck Depression Index; BFI, Brief Fatigue Inventory; CFS, Cancer Fatigue Scale; FACIT-F, Functional Assessment of Chronic Illness Therapy - Fatigue; FACT-G, Functional Assessment of Cancer Therapy - General; QOL, quality of life. daily. Of the participants analyzed in the placebo arm, 27 (87\%) took the complete study treatment. Four participants $(13 \%)$ in the placebo arm did not receive complete study treatment for the following reasons: 2 due to patient noncompliance, one stopped study treatment after day 43 due to thrombocytopenia, and one was taken off treatment on day 36 at the discretion of the treating physician.

Based on analysis of the participants with complete or nearcomplete data at baseline and day 43 , irrespective of the amount of treatment received, there were no statistically significant differences between the armodafinil arm and the placebo arm in the 42-day change in FACIT-fatigue, FACT-G, CFS, or BFI (Table 2). Indeed, there were no statistically significant differences between arms for any time point (day 22 or day $56 \mathrm{com}$ pared with baseline) for FACIT-fatigue, FACT-G, CFS, or BFI (Table 2). We also examined data for patients with more baseline fatigue (defined as a baseline FACIT-fatigue score worse than the median). There was no statistically significant difference in the 21-day, 42-day, or 55-day change from baseline in FACIT-fatigue, FACT-G, CFS, or BFI. Treatment was well tolerated with few grade 3 or 4 toxicities related to armodafinil or placebo (Table 3).

Per intention to treat approach, all patients randomized were included in the missing data analysis. Their data were imputed based on statistical approaches previously described in the methods section. There was no significant difference between the two study arms in the change in FACIT-fatigue score from baseline to day $43(P=.181)$. This result was validated by a linear mixed model fitted on all participants with at least one data point (patients without any data excluded). The $P$-value for the treatment arm was .47 and .13 for change over time by arm. The Pattern Mixture Model approach, which was tested on all participants with at least one data point, suggested that there was no difference between the arms even after accounting for the potential bias of the missing data $(P=.234)$. Full statistical inference from 100 control-based imputed datasets is presented in Appendix 1.

Table 2. Outcomes

\begin{tabular}{|c|c|c|c|c|c|c|}
\hline \multirow[t]{2}{*}{ Outcome } & \multirow[t]{2}{*}{ Time Point } & \multicolumn{2}{|c|}{ Armodafinil } & \multicolumn{2}{|c|}{ Placebo } & \multirow[t]{2}{*}{$P$ Value } \\
\hline & & $N$ & Median Change (Range) & $\mathrm{N}$ & Median Change (Range) & \\
\hline \multirow[t]{3}{*}{ FACIT-fatigue } & 21-day change (baseline vs day 22) & 31 & $-1(-22$ to 14$)$ & 31 & $0(-26$ to 22$)$ & .9 \\
\hline & 42-day change (baseline vs day 43 ) & 31 & $-1(-22$ to 48$)$ & 29 & $-3(-38$ to 22$)$ & .3 \\
\hline & 55-day change (baseline vs day 56) & 29 & $-1(-30$ to 19$)$ & 29 & $-0.5(-31$ to 14$)$ & 0.97 \\
\hline \multirow[t]{3}{*}{ FACT-G } & 21-day change (baseline vs day 22) & 31 & $-1.76(-45.01$ to 24.5$)$ & 29 & $-4.94(-45$ to 21$)$ & .5 \\
\hline & 42-day change (baseline vs day 43) & 30 & $2.50(-28$ to 25.17$)$ & 28 & $-2.30(-58.6$ to 20.17$)$ & .1 \\
\hline & 55-day change (baseline vs day 56) & 29 & $1.83(-40$ to 22$)$ & 29 & $-0.84(-24$ to 16$)$ & .3 \\
\hline \multirow[t]{3}{*}{ CFS } & 21-day change (baseline vs day 22) & 32 & $1(-22$ to 15$)$ & 31 & $0(-39$ to 25$)$ & 0.97 \\
\hline & 42-day change (baseline vs day 43) & 31 & $2(-27$ to 22$)$ & 30 & $2(-43$ to 36$)$ & .9 \\
\hline & 55-day change (baseline vs day 56) & 29 & $1(-19$ to 23$)$ & 29 & $3(-46$ to 20$)$ & .4 \\
\hline \multirow[t]{3}{*}{ BFI } & 21-day change (baseline vs day 22) & 31 & $0(-6.89$ to 7.78$)$ & 31 & $0.56(-5.11$ to 5.23$)$ & .2 \\
\hline & 42-day change (baseline vs day 43) & 31 & $0.11(-6.78$ to 6.33$)$ & 30 & $0.61(-3.45$ to 7.56$)$ & .2 \\
\hline & 55-day change (baseline vs day 56) & 29 & $-0.22(-3.11$ to 8.67$)$ & 28 & $0.33(-3.45$ to 5$)$ & .3 \\
\hline
\end{tabular}

BFI, Brief Fatigue Inventory; CFS, Cancer Fatigue Scale; FACIT-fatigue, Functional Assessment of Chronic Illness Therapy - Fatigue subscale; FACT-G, Functional Assessment of Cancer Therapy - General. 
Table 3. Adverse events at least possibly related to study treatment.

\begin{tabular}{|c|c|c|c|c|}
\hline \multirow[t]{2}{*}{ Toxicity } & \multicolumn{2}{|c|}{$\begin{array}{l}\text { Armodafinil } \\
(\mathrm{N}=42)\end{array}$} & \multicolumn{2}{|c|}{ Placebo $(\mathrm{N}=39)$} \\
\hline & Grade 3 & Grade 4 & Grade 3 & Grade 4 \\
\hline Abdominal pain & 1 & 0 & 0 & 0 \\
\hline Confusion & 0 & 0 & 1 & 0 \\
\hline Dehydration & 0 & 1 & 0 & 0 \\
\hline Diarrhea & 1 & 0 & 1 & 0 \\
\hline Glaucoma & 0 & 0 & 1 & 0 \\
\hline Hyperkalemia & 1 & 0 & 0 & 0 \\
\hline Hypokalemia & 1 & 0 & 0 & 0 \\
\hline Hypomagnesemia & 1 & 0 & 0 & 0 \\
\hline Ileus & 1 & 0 & 0 & 0 \\
\hline Insomnia & 1 & 0 & 0 & 0 \\
\hline Mood alteration (agitation) & 0 & 0 & 1 & 0 \\
\hline Obstruction, cecum & 1 & 0 & 0 & 0 \\
\hline Rash & 0 & 0 & 1 & 0 \\
\hline Seizure & 1 & 0 & 0 & 0 \\
\hline Speech impairment & 0 & 0 & 1 & 0 \\
\hline
\end{tabular}

\section{Discussion}

An 8-week course of armodafinil $150 \mathrm{mg}$ daily did not improve fatigue or QOL compared with placebo in glioma patients undergoing RT based on the change in FACIT-fatigue, FACT-G, CFS, or BFI on days 22,43 , or 56 from baseline. These findings are consistent with prior randomized studies of central nervous system (CNS) stimulants in cancer patients, including primary brain tumors ${ }^{4,14,15}$ and lung cancer. ${ }^{16}$ Page et al performed a phase 2 randomized, double-blinded, placebo-controlled study of armodafinil for primary brain tumor patients receiving brain irradiation and similarly found no statistical differences in fatigue between the armodafinil arm and the placebo arm. ${ }^{4}$ Our study differs with regard to study population (any primary brain tumor in their study vs only gliomas in our study) and study treatment length (10 weeks in their study vs 8 weeks in our study). Subgroup analysis of patients with more baseline fatigue by Page et al suggested an improvement in QOL and reduced fatigue when using armodafinil. We did not detect any improvements in QOL or fatigue at any time point in the participants with more baseline fatigue.

Several confounding factors that can contribute to fatigue include depression or other mood problems, physical disability, tumor grade, dose of radiation, whether chemotherapy is given concomitantly, and steroid use. While randomization may not completely correct for all confounders, there were no statistically significant differences between groups with respect to KPS, glioma grade, the median dose of radiation, or the percentage of patients receiving concomitant chemotherapy. We determined the severity of depression in each patient by the BDI, similarly at baseline, day 22 , day 43 , and day 56 . We did not find any statistically significant differences between groups based on the change in BDI on days 22,43 , or 56 from baseline.

Corticosteroids have been reported to improve fatigue in the general cancer population. ${ }^{17} \mathrm{~A}$ double-blind, randomized, placebo-controlled study of dexamethasone $(4 \mathrm{mg}$ twice daily for 14 days) in 84 patients with advanced cancer revealed a significant improvement in FACIT-F. ${ }^{18}$ As many glioma patients are already on dexamethasone for management of cerebral edema, it is unclear if increasing the dose of dexamethasone would be a meaningful intervention for fatigue. Our study was not designed to examine the role of steroids in fatigue. Nonetheless, we did collect information regarding dexamethasone dosing while on study. There was no significant correlation between dexamethasone dosing and FACIT-fatigue score at baseline, day 22 , day 43 , or day 56 . Dexamethasone dose over time also did not correlate with the FACIT-fatigue score.

While we did not find a statistically significant improvement in fatigue or QOL with armodafinil in glioma patients undergoing RT, there was a trend towards improvement in the 42-day change in FACIT-fatigue, FACT-G, and BFI. It is possible that armodafinil truly does improve fatigue in this patient population, but our study had low statistical power to detect a difference due to small sample size, minimal effects, or both. Other unanswered questions include whether a higher dose of armodafinil $(200 \mathrm{mg} /$ day) could be beneficial and whether patients with glioblastoma (GBM) might benefit more than patients with low-grade gliomas. Finally, our intervention was limited to 8 weeks, although the fatigue from radiation may extend beyond this time frame. It is unclear whether a longer course of armodafinil would have been more beneficial or whether armodafinil should have been administered during and/or after completing RT. There is now an ongoing randomized, placebo-controlled phase 3 trial of armodafinil for fatigue in GBM patients (who are stable post RT) that examines this higher dose of armodafinil (A221101). ${ }^{19}$

\section{Funding}

This study was supported by the National Brain Tumor Society and Cephalon, Inc., a wholly owned subsidiary of Teva Pharmaceuticals.

Conflict of interest statement. Eudocia Q. Lee: consultant for Genentech; honoraria from UptoDate. Jan Drappatz: stock in Exelixis and Bristol-Myers Squibb; honoraria from UpToDate. Eric T. Wong: research funding to his institution from Angiochem, AstraZeneca, Northwest Biotherapeutics, Novartis, Novocure, Pfizer, Plexxicon. David A Reardon: honoraria from Abbvie, Cavion, Genentech/Roche, Merck, Midatech, Momenta Pharmaceuticals, Novartis, Novocure, Regeneron, Stemline Pharmaceuticals; consultant for Cavion, Genentech/Roche, Merck, Momenta Pharmaceuticals, Novartis, Novocure, Regeneron, Stemline Therapeutics; speaker bureau for Genentech/Roche, Merck; research funding from Precision Health Economics and funding to his institution from Celldex as well as Incyte. Lakshmi Nayak: peer reviewer for UpToDate; consultant for Amgen. Mikael L. Rinne: consultant for N-of-One. Brian M. Alexander: consultant for Abbvie and Precision Health Economics. Patrick Y Wen: consultant for Abbvie, Cavion, Celldex, Genentech/Roche, Midatech, Momental Pharmaceuticals, Novartis, Novocure Sigma-Tau, Vascular Biogenics, Agios, Angiochem, GlaxoSmithKline, Karyopharm Therapeutics, Regerson, and Sanofi speaker bureau for Merck.

\section{References}

1. Armstrong TS, Gilbert MR. Practical strategies for management of fatigue and sleep disorders in people with brain tumors. Neuro Oncol. 2012;14(Suppl 4):iv65-iv72. 
2. Lovely M, Miaskowski C, Dodd M. Relationship between fatigue and quality of life in patients with glioblastoma multiformae. Oncol Nurs Forum. 1999;26(5):921-925.

3. Schiff D, Lee EQ, Nayak L, Norden AD, Reardon DA, Wen PY. Medical management of brain tumors and the sequelae of treatment. Neuro Oncol. 2015;17(4):488-504.

4. Page BR, Shaw EG, Lu L, et al. Phase II double-blind placebocontrolled randomized study of armodafinil for brain radiationinduced fatigue. Neuro Oncol. 2015;17(10):1393-401.

5. Cella DF, Tulsky DS, Gray G, et al. The Functional Assessment of Cancer Therapy scale: development and validation of the general measure. J Clin Oncol. 1993;11(3):570-579.

6. Bruera E, Valero V, Driver $L$, et al. Patient-controlled methylphenidate for cancer fatigue: a double-blind, randomized, placebo-controlled trial. J Clin Oncol. 2006;24(13):2073-2078.

7. Cella D, Lai JS, Chang CH, Peterman A, Slavin M. Fatigue in cancer patients compared with fatigue in the general United States population. Cancer. 2002;94(2):528-538.

8. Okuyama T, Akechi T, Kugaya A, et al. Development and validation of the cancer fatigue scale: a brief, three-dimensional, self-rating scale for assessment of fatigue in cancer patients. J Pain Symptom Manage. 2000;19(1):5-14.

9. Mendoza TR, Wang XS, Cleeland CS, et al. The rapid assessment of fatigue severity in cancer patients: use of the Brief Fatigue Inventory. Cancer. 1999;85(5):1186-1196.

10. Rubin DB, Schenker N. Multiple imputation in health-care databases: an overview and some applications. Stat Med. 1991; 10(4):585-598.

11. Little R, Yau L. Intent-to-treat analysis for longitudinal studies with drop-outs. Biometrics. 1996;52(4):1324-1333.

12. Ratitch B, O'Kelly M. Implementation of Pattern-Mixture Models Using Standard SAS/STAT Procedures. Paper presented at: PharmaSUG Annual Conference; 8-11 May 2011; Nashville, TN.

13. National Research Council (US) Panel on Handling Missing Data in Clinical Trials. The Prevention and Treatment of Missing Data in Clinical Trials. Washington, DC: National Academies Press; 2010. Available from: http://www.ncbi.nlm.nih.gov/books/NBK209904/. doi: 10.17226/12955.

14. Boele FW, Douw L, de Groot M, et al. The effect of modafinil on fatigue, cognitive functioning, and mood in primary brain tumor patients: a multicenter randomized controlled trial. Neuro Oncol. 2013;15(10):1420-1428.

15. Butler JM Jr., Case LD, Atkins J, et al. A phase III, double-blind, placebo-controlled prospective randomized clinical trial of d-threo-methylphenidate $\mathrm{HCl}$ in brain tumor patients receiving radiation therapy. Int J Radiat Oncol Biol Phys. 2007;69(5): $1496-1501$.

16. Spathis A, Fife K, Blackhall F, et al. Modafinil for the treatment of fatigue in lung cancer: results of a placebo-controlled, double-blind, randomized trial. J Clin Oncol. 2014;32(18): $1882-1888$.

17. de Raaf PJ, van der Rijt CC. Can you help me feel less exhausted all the time? J Clin Oncol. 2013;31(25):3056-3060.

18. Yennurajalingam S, Frisbee-Hume S, Palmer JL, et al. Reduction of cancer-related fatigue with dexamethasone: a double-blind, randomized, placebo-controlled trial in patients with advanced cancer. J Clin Oncol. 2013;31(25):3076-3082.

19. Alliance for Clinical Trials in Oncology; National Cancer Institute. Armodafinil in Reducing Cancer-Related Fatigue in Patients With High Grade Glioma. 2013-[cited 2015 July 15]. Available from: http://clinicaltrials.gov/show/NCT01781468 NLM Identifier: NCT01781468; https://clinicaltrials.gov/ct2/show/NCT01781468. Accessed July 15, 2015. 